\title{
INCIDENCIA DE LA COCHINILLA ROSADA DEL HIBISCO EN CULTIVARES DE MANGO DE NAYARIT, MÉXICO
}

\author{
Ninfa M. Rosas-García ${ }^{1}$ \& Gaspar M. Parra-Bracamonte ${ }^{2}$ \\ ${ }^{1}$ Laboratorio de Biotecnología Ambiental, Centro de Biotecnología Genómica-IPN, Blvd. del Maestro \\ s/n, Col. Narciso Mendoza, 88710 Reynosa, Tamp., México. <nrosas@ipn.mx> \\ ${ }^{2}$ Laboratorio de Biotecnología Animal, Centro de Biotecnología Genómica-IPN, Blvd. del Maestro s/n, \\ Col. Narciso Mendoza, 88710 Reynosa, Tamp., México. <gparra@ipn.mx>
}

Rosas-García, N. M. \& G. M. Parra-Bracamonte. 2011. Incidencia de la cochinilla rosada del hibisco en cultivares de mango de Nayarit, México. Acta Zoológica Mexicana (n. s.), 27(2): 407-418.

RESUMEN. La incidencia de la cochinilla rosada del hibisco (CRH), Maconellicoccus hirsutus (Green) (Hemiptera: Pseudococcidae) fue evaluada en seis cultivares de mango en producción en Bahía de Banderas estado de Nayarit, México. La presencia de esta plaga fue determinada mediante el monitoreo de cada etapa de desarrollo, ninfas de primer instar o caminantes (N1), ninfas de segundo instar (N2), ninfas de tercer instar (N3), hembras (H) y ovisacos (O) en los cultivares de mango, Tommy Atkins, Haden, Manila, Ataulfo, Keitt, y Kent. Además, la presencia del insecto se determinó en yemas terminales y frutos (en los árboles) y en malezas aledañas. El estudio indicó que la incidencia mensual promedio de individuos $\mathrm{N} 1$ y N2 fue mayor entre los meses de enero y febrero, mayo y junio, y agosto y septiembre, mientras que para individuos N3 ocurrió entre julio y agosto. Todas las etapas de desarrollo de la CRH presentaron la mayor incidencia en el cultivar Ataulfo. La media de individuos N1 y N2 en los frutos y en la maleza no mostró diferencia significativa $(\mathrm{P}>0.05)$, pero la media de individuos $\mathrm{N} 3$, hembras y ovisacos presentes en fruto fue mayor a la media de individuos observados en las yemas terminales y en la maleza. Los resultados sugieren la existencia de una relación entre las diferentes etapas de desarrollo de la plaga, el cultivar y la fenología del árbol, en donde se observa una mayor incidencia de los primeros instares por el cultivar Ataulfo y una mayor incidencia a partir de la etapa N3 por el fruto. Es importante conocer la susceptibilidad de los cultivares de mango al ataque de la $\mathrm{CRH}$ para poder ejercer un manejo que evite la diseminación de la plaga.

Palabras clave: Insecto polífago, monitoreo, Maconellicoccus hirsutus, fruto de mango, ninfas.

Rosas-García, N. M. \& G. M. Parra-Bracamonte. 2011. Incidence of the pink hibiscus mealybug in mango cultivars from Nayarit, Mexico. Acta Zoológica Mexicana (n. s.), 27(2): 407-418.

ABSTRACT. The incidence of the pink hibiscus mealybug (PHM) Maconellicoccus hirsutus (Green) (Hemiptera: Pseudococcidae) was evaluated in six cultivars of mango grown in Bahía de Banderas state of Nayarit, México. The incidence of the pest was determined by monitoring each developmental stage, first instar nymphs or crawlers (N1), second instar nymphs (N2), third instar nymphs (N3), females (F), and ovisacs (O) on Tommy Atkins, Haden, Manila, Ataulfo, Keitt, and Kent cul-

Recibido: 03/08/2010; aceptado: 01/02/2011. 
tivars. Presence of insects was also determined in three different locations: terminal buds and fruits in trees, as well as surrounding weeds. Results indicated that average monthly incidence of N1 and N2 individuals was higher between January and February, May and June, and August and September, incidence of N3 individuals was also higher between July and August. All developmental stages showed the highest incidence in cultivar Ataulfo. Mean number of N1 and N2 individuals on fruits and weeds did not show significant difference, but mean number of N3 individuals, females and ovisacs on fruits was higher with respect to individuals on buds and weeds. Results suggest an interaction among the developmental stages, cultivars and tree phenology. A preference of the first instars for cultivar Ataulfo was observed, as well as a preference of N3 individuals for fruits. It is important to assess the susceptibility of mango cultivars to PHM attack in order to perform a suitable control to avoid pest dissemination.

Key words: Polyphagous insect, monitoring, Maconellicoccus hirsutus, mango fruit, nymphs.

\section{INTRODUCCIÓN}

Maconellicoccus hirsutus (Green) (Hemiptera: Pseudococcidae) comúnmente conocida como "la cochinilla rosada del hibisco" (CRH), es una plaga que fue descrita originalmente en la India (Cermeli et al. 2002). Esta plaga se dispersó a todo el sureste asiático y parte de China y posteriormente en 1908 se detectó en Egipto, desde donde se dispersó hacia la mayoría de los países africanos (Williams 1996). Su presencia en Australia se reportó desde 1959 (Williams 1986) y en 1983 se introdujo al Hemisferio Occidental por Hawaii. En 1994 se introdujo en Granada, desde donde se dispersó a otras regiones del Caribe como Trinidad y Tobago, Guyana, St. Martin, Curazao, St. Lucia, St. Kitts y Venezuela (Williams 1996). En 1999 se detectó en Belice (Sosa 2001) y en California y en 2002 en Florida (Roltsch et al. 2004). En México se detectó por primera vez en 1999 en Mexicali, Baja California y en 2004 se confirmó su presencia en Bahía Banderas, Nayarit y en los Estados de Quintana Roo y Yucatán (SAGARPA 2004, NAPPO 2004, http://www.pestalert.org/viewArchNewsStory. $\mathrm{cfm}$ ?nid=297). Actualmente está presente en más de 55 países tropicales y subtropicales (Kairo et al. 2000).

La CRH es una plaga polífaga de importancia económica y cuarentenaria para la agricultura mundial; que ataca a más de 350 especies de plantas (Cermeli et al. 2002) distribuidas en 218 géneros y 70 familias botánicas (Padilla 2000), entre las que se encuentran árboles forestales, frutales, ornamentales, tubérculos, vegetales y hortalizas entre otras, afectando en tronco, yemas terminales, nudos, frutos e inclusive debajo de la corteza (Mani 1988, Vázquez 1997) e incluso en la raíz (Ghose 1972, Rao \& Srinivasan 1987).

$\mathrm{Su}$ eficiencia como insectos chupadores se debe a su fina probóscide que penetra el tejido de la planta hospedera, creando una fuerza con la que los fluidos son succionados o inyectados a través de sus canales salivarios. Este sistema le facilita la absorción de agua y azúcares, los cuales excreta rápidamente en forma de mielecilla (Grimaldi \& Engel 2005). El proceso de alimentación causa malformaciones en los 
frutos, hojas y yemas terminales, reduce el crecimiento, disminuye la producción de frutos y finalmente causa la muerte de la planta.

En México el árbol del mango (Mangifera indica L.) es frecuentemente atacado por diversas plagas de insectos, como la mosca de la fruta, trips, chicharritas, escamas, hormigas, barrenadores del tronco y ramas (Chávez-Contreras et al. 2001, Ramos Serrano et al. 2008) y recientemente por la CRH. Debido a que el estado de Nayarit es uno de los principales estados productores de mango a nivel nacional, la presencia de la $\mathrm{CRH}$ ha limitado la producción y comercialización del fruto causando importantes pérdidas a los productores (Ramos-Novelo 2003). En el año de 2008 la zona bajo control fitosanitario en México se extendió y abarcó, los municipios de Bahía de Banderas, Compostela, Ruiz, Santiago, Tuxpan, Rosamorada, Tecuala y Acaponeta en el Estado de Nayarit, así como los de Puerto Vallarta, Tomatlán y Cihuatlán en el Estado de Jalisco (Diario Oficial de la Federación 2007).

La CRH está considerada como una plaga de alta peligrosidad debido a su polifagia y el ataque que ocasiona puede relacionarse con algunas partes de la planta de consistencia jugosa o azucarada o que le brinden protección para su desarrollo. Por lo tanto, la incidencia de la CRH en el cultivo de mango puede variar en los diferentes cultivares, así como en algunas partes de la planta o malezas aledañas que le sirvan para su alimentación y desarrollo. En base a estas consideraciones, el objetivo del presente trabajo fue determinar la incidencia de la CRH en seis cultivares de mango en producción y malezas asociadas en la zona de Bahía de Banderas Nayarit.

\section{MATERIAL Y MÉTODOS}

Monitoreo de M. hirsutus. El estudio se realizó de enero a diciembre de 2005 en 18 huertas de mango de temporal y de riego localizadas en 6 ejidos del municipio de Bahía de Banderas en el estado de Nayarit, México, abarcando una superficie de 73.5 ha (Fig. 1). Los puntos de monitoreo fueron posicionados utilizando un GPS (Magellan Meridian ${ }^{\circledR}$ Color, Thales Navigation, San Dimas, CA), y con ellos se realizó un mapa utilizando el programa ArcView GIS versión 3.3.

Se seleccionaron seis tipos de cultivares, Tommy Atkins, Haden, Manila, Ataulfo, Keitt, y Kent y se consideraron únicamente las huertas que tenían un sólo tipo de cultivar sembrado (Cuadro 1). Las etapas de desarrollo de la CRH consideradas en el estudio fueron: ninfas de primer instar o caminantes (N1), ninfas de segundo instar (N2), ninfas de tercer instar (N3), hembras (H) y ovisacos (O). Las estructuras de los árboles muestreados fueron yemas terminales y frutos, también se muestrearon las malezas aledañas a los árboles.

El procedimiento de muestreo en cada huerta se realizó una vez por semana siguiendo un patrón en zig-zag de acuerdo al número de has que constituía la huerta. Para el muestreo en árboles de mango se tomó un árbol como referencia y se siguió 


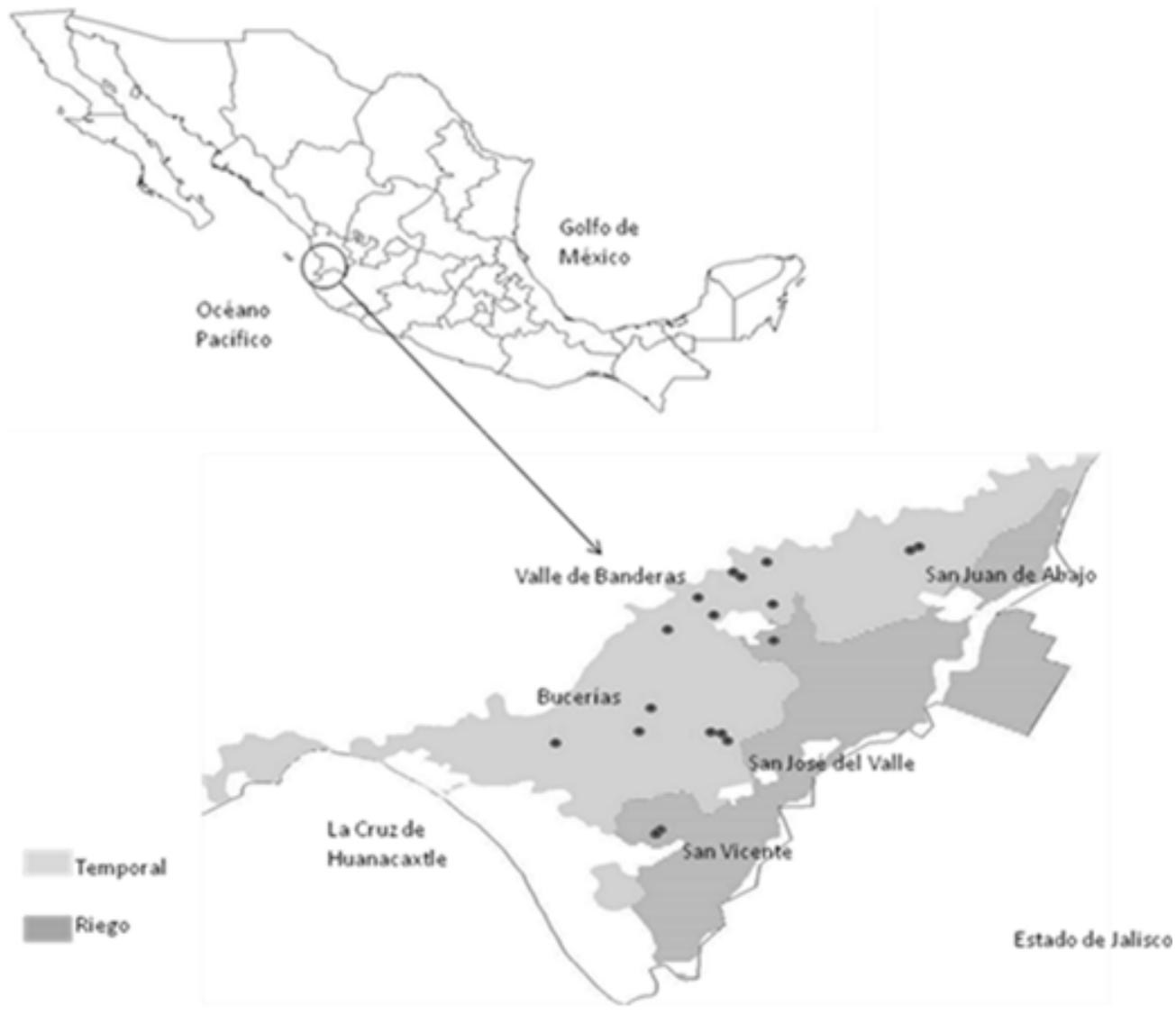

Figura 1. Sitios de monitoreo de la CRH en huertas de mango de la región de Bahía de Banderas del estado de Nayarit, México.

una trayectoria en línea recta hasta llegar al punto de muestreo. A partir de este punto se siguió una trayectoria en ángulo de $45^{\circ}$ (diagonal) hasta llegar al siguiente punto de muestreo (Fig. 2). El punto de muestreo correspondió al quinto árbol de la trayectoria recorrida. El número de árboles muestreados en base al número de has se indica en el Cuadro 2. Cada ha estaba constituida por 144 árboles de mango distribuidos en 12 surcos. Cada árbol muestreado constituyó una unidad experimental y la zona de muestreo en cada unidad experimental se consideró desde la base del árbol a nivel del suelo hasta una altura de $2 \mathrm{~m}$.

Se seleccionaron cuatro yemas terminales de $5 \mathrm{~cm}$ de longitud (uno por cada punto cardinal) por árbol. Para los árboles con frutos se muestrearon dos yemas termina- 
Cuadro 1. Huertas de mango seleccionadas para la evaluación de la presencia de la CRH en el estado de Nayarit, México.

\begin{tabular}{cllll}
\hline Registro Huerta & Ejido & Cultivar & Coordenadas & Has \\
\hline 1 & Valle de Banderas & Tommy Atkins & N20 48 19.0 W105 16 05.3 & 4.00 \\
2 & Valle de Banderas & Tommy Atkins & N20 49 00.2 W105 15 36.2 & 4.00 \\
3 & Valle de Banderas & Tommy Atkins & N20 48 38.3 W105 15 21.0 & 2.00 \\
4 & Valle de Banderas & Haden & N20 49 27.1 W105 14 55.0 & 1.00 \\
5 & El Porvenir & Haden & N20 45 53.2 W105 17 52.0 & 4.00 \\
6 & El Porvenir & Haden & N20 46 08.4 W105 16 32.5 & 8.00 \\
7 & Valle de Banderas & Ataulfo & N20 48 51.7 W105 14 24.6 & 5.00 \\
8 & Valle de Banderas & Ataulfo & N20 43 55.9 W105 16 16.7 & 2.00 \\
9 & Valle de Banderas & Ataulfo & N20 48 04.8 W105 14 24.2 & 1.00 \\
10 & San José & Manila & N20 46 05.2 W105 15 14.0 & 4.00 \\
11 & San Juan de Abajo & Manila & N20 50 01.6 W105 12 13.6 & 7.00 \\
12 & Valle de Banderas & Manila & N20 49 46.3 W105 14 30.9 & 1.50 \\
13 & San José del Valle & Kent & N20 46 37.7 W105 16 21.5 & 1.00 \\
14 & San Vicente & Kent & N20 46 07.2 W105 15 24.4 & 10.00 \\
15 & San Vicente & Kent & N20 45 55.8 W105 15 08.1 & 8.00 \\
16 & San Juan de Abajo & Keitt & N20 50 05.7 W105 12 05.1 & 4.00 \\
17 & Valle de Banderas & Keitt & N20 44 01.7 W105 16 11.3 & 2.00 \\
18 & Valle de Banderas & Keitt & N20 49 33.6 W105 15 02.7 & 5.00 \\
\hline
\end{tabular}

les y cuatro frutos (incluyendo el pedúnculo). Para el muestreo en la maleza aledaña se seleccionaron 3 sitios por cada lado de la huerta en los límites externos, para tener un total de 12 sitios. En los sitios seleccionados se determinó la presencia del insecto en las malezas encontradas en un radio de $2 \mathrm{~m}$. En todos los casos el monitoreo se realizó en forma visual, registrando el número de individuos de la CRH y su etapa de desarrollo.

La incidencia estacional de la CRH se ajustó a una curva de tendencia mensual utilizando los promedios de incidencia estimados (SAS, 2001).

Posteriormente, los datos de incidencia se analizaron con la prueba no paramétrica de Kruskal-Wallis para determinar los niveles de significancia de la incidencia de la plaga con respecto a los cultivares de mango, a la parte del árbol o maleza atacadas. Complementariamente, fueron realizadas las comparaciones pareadas para determinar diferencias puntuales en los mismos factores con respecto a la incidencia de la plaga utilizando la misma prueba en el paquete estadístico SAS ver. 8.20 (SAS 2001). 


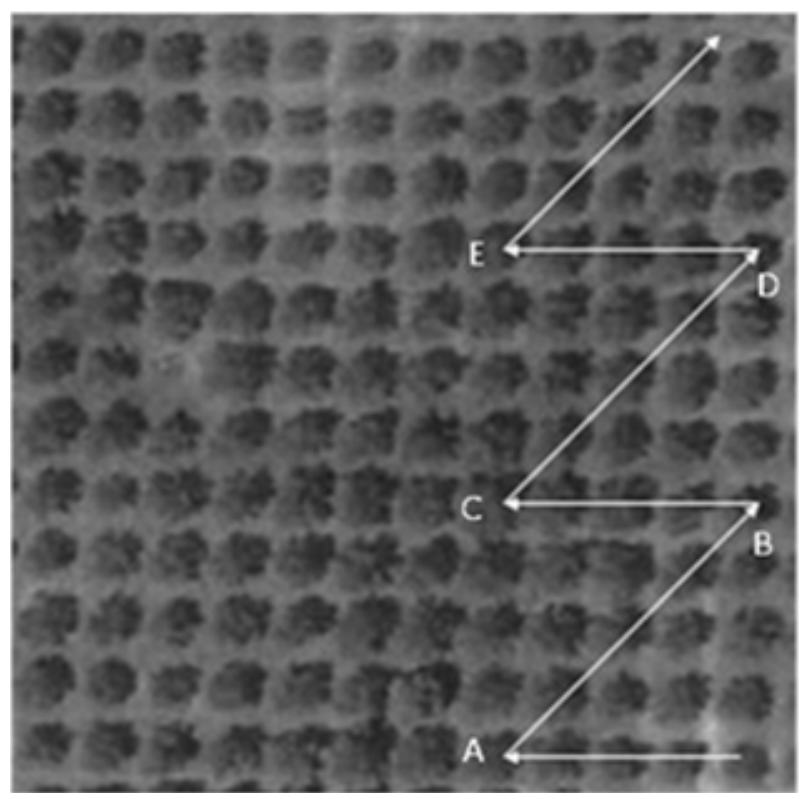

Figura 2. Imagen de la vista aérea de una hectárea sembrada con mango (144 árboles) en el estado de Nayarit, en la cual se indica el muestreo en zig-zag para el monitoreo de la CRH. Los puntos A, B, C, D y E indican los puntos de muestreo.

Cuadro 2. Número de árboles de mango a muestrear según el tamaño de la huerta.

\begin{tabular}{cc}
\hline Número de hectáreas & Número de árboles a muestrear \\
\hline 0.1 a 1.0 & 15 \\
1.1 a 2.0 & 20 \\
2.1 a 3.0 & 25 \\
3.1 a 4.0 & 30 \\
Mayor a 4.1 & 35 \\
\hline
\end{tabular}

\section{RESULTADOS}

La incidencia de la CRH durante 2005 en los cultivares de mango en el área de Bahía de Banderas, Nayarit, indicó que la incidencia mensual promedio de N1 fue mayor entre los meses de enero y febrero, mayo y junio, y agosto y septiembre. La incidencia de individuos N2 ocurrió con un patrón similar al de los individuos N1 y la mayor incidencia de individuos N3 se observó entre julio y agosto, sin embargo su incidencia fue menor durante todo el año con respecto a los individuos N1 y N2 (Fig. 3). La incidencia de las hembras y ovisacos fue menor a 2 individuos durante todo el año (Fig. 4). 


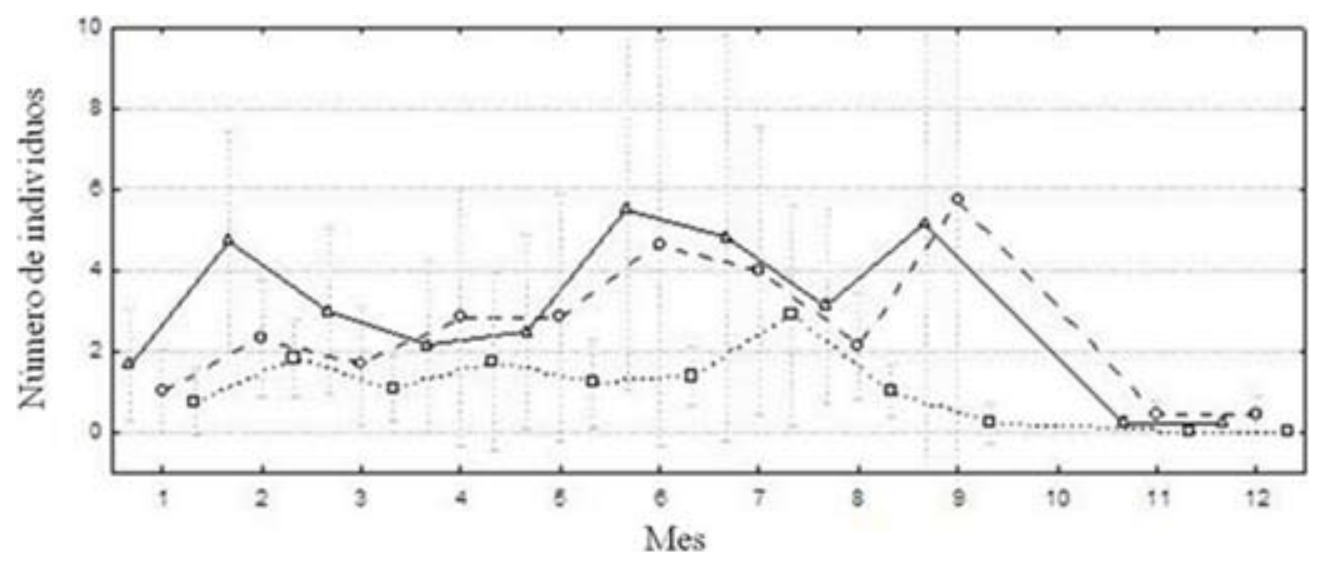

Figura 3. Patrón de la incidencia mensual promedio de las etapas de desarrollo N1, N2 y N3 de la CRH en cultivares de mango sembrado en el estado de Nayarit México durante $2005(\Delta=\mathrm{N} 1, \mathrm{o}=\mathrm{N} 2$, $\square=\mathrm{N} 3$ ).

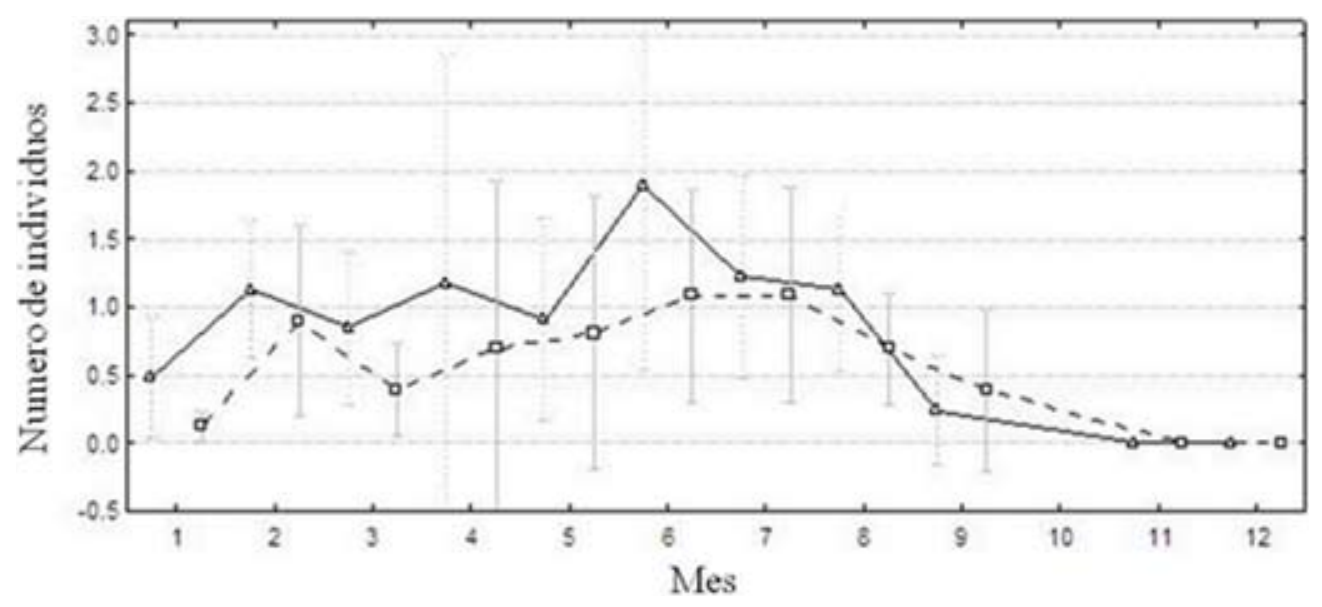

Figura 4. Patrón de la incidencia mensual promedio de hembras y ovisacos de la CRH en cultivares de mango sembrado en el estado de Nayarit México durante 2005 ( $\Delta=$ hembras, $\square=$ Ovisaco).

Todas las etapas de desarrollo de la CRH presentaron la mayor incidencia en el cultivar Ataulfo (Cuadro 4). Los cultivares Ataulfo y Keitt presentaron las medias más altas de las N1 y N2 de la CRH y que fueron significativamente diferentes a las medias que se presentaron en el resto de los cultivares de mango (Cuadro 3). Durante 
Rosas-García \& Parra-Bracamonte: Incidencia de la cochinilla rosada en mango

Cuadro 3. Niveles de significancia y estadísticos del análisis no paramétrico Kruskal-Wallis de la incidencia de la $\mathrm{CRH}$ en seis cultivares de mango y en tres ubicaciones de muestreo en huertas de Nayarit México.

\begin{tabular}{lcccccc}
\hline & \multicolumn{3}{c}{ Por cultivar } & \multicolumn{3}{c}{ Por ubicación de muestreo* } \\
\hline Factor & $p$ & $H$ & $C$ & $p$ & $H$ & $C$ \\
\hline N1 & 0.0115 & 12.9 & 0.87 & $<0.0001$ & 23.72 & 0.87 \\
N2 & 0.0009 & 17.79 & 0.86 & $<0.0001$ & 49.55 & 0.86 \\
N3 & 0.6613 & 1.99 & 0.61 & $<0.0001$ & 44.69 & 0.61 \\
Hembra & 0.6936 & 1.99 & 0.65 & $<0.0001$ & 62.54 & 0.65 \\
Ovisaco & 0.0812 & 5.30 & 0.54 & $<0.0001$ & 38.85 & 0.54 \\
\hline
\end{tabular}

$p$ : nivel de probabilidad, $H$ : Estadístico de prueba, $C$ : factor de corrección en caso de empates.

*Yema terminal, fruto y maleza.

Cuadro 4. Incidencia de las etapas de desarrollo de la CRH en seis cultivares de mango en el estado de Nayarit México.

\begin{tabular}{lcccccc}
\hline Cultivar & $\mathrm{N}$ & \multicolumn{5}{c}{ Número medio de individuos $\pm \mathrm{EE}^{*}$} \\
\hline Tommy Atkins & 81 & $1.18 \pm 1.19^{\mathrm{a}}$ & $1.37 \pm 1.08^{\mathrm{ab}}$ & $0.91 \pm 0.52^{\mathrm{ab}}$ & $0.56 \pm 0.33^{\mathrm{ab}}$ & $0.39 \pm 0.27^{\mathrm{a}}$ \\
Haden & 75 & $4.23 \pm 1.22^{\mathrm{a}}$ & $2.21 \pm 1.13^{\mathrm{a}}$ & $1.70 \pm 0.55^{\mathrm{ab}}$ & $0.56 \pm 0.34^{\mathrm{ab}}$ & $0.50 \pm 0.28^{\mathrm{b}}$ \\
Ataulfo & 63 & $5.42 \pm 1.33^{\mathrm{bc}}$ & $6.41 \pm 1.23^{\mathrm{bc}}$ & $2.57 \pm 0.60^{\mathrm{b}}$ & $2.11 \pm 0.37^{\mathrm{b}}$ & $1.8 \pm 0.31^{\mathrm{b}}$ \\
Manila & 75 & $3.73 \pm 1.22^{\mathrm{acd}}$ & $2.68 \pm 1.13^{\mathrm{acd}}$ & $0.86 \pm 0.55^{\mathrm{ab}}$ & $0.87 \pm 0.34^{\mathrm{ab}}$ & $0.44 \pm 0.28^{\mathrm{a}}$ \\
Kent & 82 & $1.46 \pm 1.17^{\mathrm{a}}$ & $0.85 \pm 1.08^{\mathrm{a}}$ & $0.83 \pm 0.52^{\mathrm{a}}$ & $1.00 \pm 0.32^{\mathrm{a}}$ & $0.17 \pm 0.27^{\mathrm{a}}$ \\
Keitt & 74 & $4.20 \pm 1.23^{\mathrm{bd}}$ & $3.11 \pm 1.13^{\mathrm{bd}}$ & $1.54 \pm 0.55^{\mathrm{ab}}$ & $1.17 \pm 0.34^{\mathrm{ab}}$ & $0.97 \pm 0.28^{\mathrm{ab}}$ \\
\hline
\end{tabular}

*Valores seguidos de letras iguales no tienen diferencia significativa.

el año 2005 la media de individuos de los instares ninfales fue menor a seis, mientras que el número de hembras y ovisacos fue menor a dos. Los individuos N3, hembras y ovisacos no presentan diferencias en todos los cultivares. Se observó que la media del número de individuos disminuye conforme el insecto se desarrolla y que ninguna etapa de desarrollo tuvo una supervivencia superior al $33 \%$ en los diferentes cultivares.

Existieron diferencias significativas en todas las etapas de desarrollo del insecto con respecto a la parte de la planta atacada como las yemas y los frutos y en la maleza aledaña $(\mathrm{P}<0.0001)$. Las medias de individuos N1 y N2 en los frutos y en la maleza no presentaron diferencia estadística, sin embargo, las medias de individuos $\mathrm{N} 3 \mathrm{hem}$ bras presentes en fruto fue mayor $(\mathrm{P}<0.0001)$ al número de individuos observados en las yemas terminales y en la maleza (Cuadro 5). 
Cuadro 5. Incidencia de las etapas de desarrollo de la CRH en tres ubicaciones diferentes en las huertas de mango del estado de Nayarit México.

\begin{tabular}{lcccccc}
\hline $\begin{array}{l}\text { Localización } \\
\text { del insecto }\end{array}$ & $\mathrm{N}$ & \multicolumn{5}{c}{ Número medio de individuos $\pm \mathrm{EE}^{*}$} \\
\hline & & $\mathrm{N} 1$ & $\mathrm{~N} 2$ & $\mathrm{~N} 3$ & $\mathrm{H}$ & $\mathrm{O}$ \\
\hline Yemas terminales & 150 & $1.54 \pm 0.86^{\mathrm{a}}$ & $0.60 \pm 0.80^{\mathrm{a}}$ & $0.43 \pm 0.38^{\mathrm{a}}$ & $0.38 \pm 0.24^{\mathrm{b}}$ & $0.16 \pm 0.20^{\mathrm{a}}$ \\
Fruto & 150 & $6.16 \pm 0.86^{\mathrm{b}}$ & $5.55 \pm 0.79^{\mathrm{b}}$ & $3.06 \pm 0.38^{\mathrm{c}}$ & $2.33 \pm 0.24^{\mathrm{c}}$ & $1.60 \pm 0.20^{\mathrm{b}}$ \\
Maleza & 150 & $2.06 \pm 0.86^{\mathrm{b}}$ & $1.72 \pm 0.72^{\mathrm{b}}$ & $0.58 \pm 0.38^{\mathrm{b}}$ & $0.32 \pm 0.24^{\mathrm{a}}$ & $0.27 \pm 0.20^{\mathrm{a}}$ \\
\hline
\end{tabular}

$\mathrm{N}=450$. § Yemas terminales y fruto en el árbol, maleza aledaña a los árboles. *Valores seguidos de letras iguales no tienen diferencia significativa.

\section{DISCUSIÓN}

La CRH tiene un amplio rango de hospederos entre los que destaca el mango (Cermelli et al. 2002, Hall et al. 2008, Hoy et al. 2008). La incidencia de esta plaga en el cultivo de mango no ha sido evaluada en México por lo que es necesario determinar su presencia y peligrosidad ya que este cultivo genera importantes divisas para el país que ocupa el cuarto lugar en producción a nivel mundial. La presencia de una plaga puede causar fuertes impactos económicos directos o indirectos. Los impactos directos incluyen los costos de prevención, control, monitoreo y daños a las especies hospederas, mientras que los impactos indirectos incluyen pérdidas económicas ocasionadas por las cuarentenas, pérdidas en el comercio debido a interrupciones en el abastecimiento y las barreras comerciales que restringen las exportaciones para evitar la introducción y esparcimiento de la plaga (Ranjan 2006). La presencia de la CRH en los cultivos de mango en el estado de Nayarit puede causar un fuerte impacto económico directo, ya que la mayor incidencia de la plaga se observó en el cultivar Ataulfo, que son árboles semi-vigorosos, muy productivos y poco alternantes, cuya época de producción ocurre de mayo a julio (Chávez-Contreras et al. 2001). Este cultivar ha reemplazado paulatinamente a otras variedades de acuerdo a las características de las zonas productivas y a las preferencias del mercado, lo que puede implicar un riesgo grave de infestación si este cultivar se distribuye ampliamente y no se toman las medidas de control pertinentes contra esta plaga.

La mayor incidencia de la plaga en todos sus estadios ocurrió durante la "temporada de mango" reportada para cada cultivar en el estado de Nayarit, que en conjunto abarca desde febrero hasta finales de septiembre y que coincide fuertemente con la producción del fruto (http://www.sagarpa.gob.mx/agricultura/Publicaciones/SistemaProducto/Lists/Mango/Attachments/1/prn_mango.pdf).

Por otra parte, las yemas terminales, frutos y malezas que actúan como sitios de posicionamiento de la plaga, ejercen una influencia significativa para la distribución 
de las diferentes etapas de desarrollo de la CRH. Las etapas de desarrollo N1 y N2 muestran la misma incidencia en fruto y en maleza, posiblemente porque son individuos más pequeños que caminan rápidamente en busca de lugares donde posarse y obtener su alimento. Sin embargo, las N3, las hembras y los ovisacos se encuentran preferentemente en el fruto. Los individuos N3 se fijan a la planta para alimentarse y desarrollarse por lo que no resulta sorprendente que su incidencia sea mayor en el fruto que es una estructura vegetativa carnosa, de pulpa jugosa y dulce. Los resultados sugieren la existencia de una interacción entre las diferentes etapas de desarrollo de la plaga, el cultivar y la fenología del árbol, en donde se observa una preferencia de los primeros instares por el cultivar Ataulfo, y una preferencia mayor por el fruto a partir de la etapa N3, lo que causa variaciones en la incidencia de la plaga.

No se observa ninguna interacción competitiva entre las etapas de desarrollo de las ninfas en donde los últimos instares puedan desplazar a los primeros instares del sitio de alimentación. Más bien se observa que los últimos instares no fueron capaces de establecerse de manera adecuada en el cultivo, pues el número de individuos encontrado es muy bajo. Básicamente se observa una alta mortalidad que ocurre en los individuos conforme crecen, aunque estos resultados pueden ser debidos a ciertos factores que ejerzan algún tipo de control. Para esta situación es importante realizar estudios posteriores que analicen la mortalidad de esta plaga en un cultivo susceptible.

La maleza se considera de importancia económica ya que compite con el cultivo por el agua, los nutrientes, la luz y el espacio, además es agresiva, tiene alta eficiencia reproductiva y sobrevivencia por varios años y son hospederas de plagas y enfermedades (Chávez-Contreras et al. 2001) por lo que sirven como un reservorio para la $\mathrm{CRH}$. Estas características aumentan los costos del cultivo y reducen el rendimiento y la calidad del fruto y la maleza infestada puede causar brotes en cultivos cercanos, por lo que el uso de herbicidas reduciría potencialmente la fuente de infestación (Chávez-Contreras et al. 2001, Pandey \& Johnson 2006). Los resultados indican que la incidencia de los individuos N1 y N2 en la maleza es similar a la del fruto, pero la incidencia disminuye a partir de los individuos en etapa N3. Así mismo es evidente que la incidencia de la plaga es muy baja en las yemas terminales, lo que indica que la plaga prefiere alimentarse de otras estructuras jugosas y dulces. De acuerdo a Lauzière \& Sétamou (2009) es importante considerar que el tiempo de desarrollo de los individuos es afectado significativamente por las plantas de las cuales se alimentan. Los nutrientes que proporciona la planta así como la textura de algunas de sus partes (características que varían entre las plantas hospederas), parecen jugar un papel significativo en el desarrollo de las ninfas. Este mismo comportamiento se ha reportado en la chinche opaca de las plantas (Lygus lineolaris) (Rhainds \& English-Loeb 2003), que al igual que la CRH es un insecto chupador. Así mismo la susceptibilidad de las plantas hospederas puede variar con respecto a los cultivares como ocurre con el hibisco, hospedero Hibiscus rosa-sinensis, preferido de M. hirsutus, sin embargo la 
plaga puede reproducirse en un cultivar susceptible que no muestre síntomas de daño (Vitullo et al. 2009).

En el estudio realizado en los cultivares de mango, los frutos del cultivar Ataulfo presentaron la mayor incidencia de la plaga lo que sugiere que este insecto muestra una preferencia por este cultivar para alimentarse, así mismo los frutos presentan una mayor incidencia de la plaga que las yemas terminales o malezas debido a su dulzura y jugosidad.

Es importante conocer la susceptibilidad de los cultivares al ataque de la $\mathrm{CRH}$ para poder ejercer un control que evite la diseminación de la plaga y en el caso del Ataulfo que es un cultivar con un fuerte potencial para la exportación, tomar las medidas de control necesarias para su producción. Por otra parte es importante considerar el manejo de las variedades menos susceptibles que ofrezcan mayores ventajas para la comercialización y menos implicaciones en costos de producción.

AGRADECIMIENTOS. La Dra. Rosas-García agradece el excelente apoyo técnico del personal del Comité Estatal de Sanidad Vegetal del Estado de Nayarit, especialmente a Néstor Isiordia y Enrique Mora y al Fondo Sectorial CONAFOR-CONACYT, proyecto No. C04-17 por el financiamiento otorgado para el desarrollo de este estudio, a la SIP y a la COFAA.

\section{LITERATURA CITADA}

Cermelli, M., P. Morales Valles, F. Godoy, R. Romero \& O. Cárdenas. 2002. Presencia de la cochinilla rosada de la cayena Maconellicoccus hirsutus (Green) (Hemiptera: Pseudococcidae) en Venezuela. Entomotropica, 1: 103-105.

Chávez-Contreras, X., A. Vega-Piña, L. M. Tapia-Vargas \& M. A. Miranda-Salcedo. 2001. Mango, su manejo y producción en el trópico seco de México. Libro Técnico Num. 1. Campo Experimental Valle de Apatzingán. CIRPAC. INIFAP. México.

Diario Oficial de la Federación. 2007. Acuerdo por el que se instrumenta el Dispositivo Nacional de Emergencia en los términos del artículo 46 de la Ley Federal de Sanidad Vegetal, con el objeto de controlar y mitigar el riesgo de dispersión de la cochinilla rosada del hibisco (Maconellicoccus hirsutus) en México. 31 de Diciembre 2007. Poder Ejecutivo - Secretaria de Agricultura, Ganadería, Desarrollo Rural, Pesca y Alimentación.

Ghose, S. K. 1972. Biology of the mealybug Maconellicoccus hirsutus. Indian Agriculture, 16: 323332.

Grimaldi, D. \& M.S. Engel. 2005. Evolution of the insects. Cambridge University Press. USA.

Hall, D.G., A. Roda, S.L. Lapointe \& K. Hibbard. 2008. Phenology of Maconellicoccus hirsutus (Hemiptera: Pseudococcidae) in Florida based on attraction of adult males to pheromone traps. Florida Entomologist, 91: 305-310.

Hoy, M. A., A. Hamon \& R. Nguyen. 2008. Pink hibiscus mealybug, Maconellicoccus hirsutus (Green). University of Florida IFAS Extension. EENY-029.

Kairo, M. T. K., G. V. Pollard, D. D. Peterkin \& V. F. Lopez. 2000. Biological control of the hibiscus mealybug, Maconellicoccus hirsutus in the Caribbean. Integrated Pest Management Reviews, 5: 241-254. 
Lauzière, I. \& M. Sétamou. 2009. Suitability of different host plants for oviposition and development of Homalodisca vitripennis (Hemiptera: Cicadellidae) and its implication on mass rearing. Annals of Entomological Society of America, 102: 642-649.

Mani, M. 1988. Bioecoloy and management of grapevine mealybug. India Institute of Horticultural Research Technical Bulletin, 5: 1-3.

NAPPO (North American Plant Protection Organization). 2004. Detection of pink hibiscus mealybug (Maconellicoccus hirsutus Green), in the municipality of Bahia de Banderas in the State of Nayarit, Mexico. Archived News Stories. http://www.pestalert.org/viewArchNewsStory.cfm?nid=297.

Padilla, M. R. 2000. Bioecología de la cochinilla rosada y su riesgo de ingreso en Honduras. Manejo Integrado de Plagas, 57: 10-22.

Pandey, R. R. \& M. W. Johnson. 2006. Weeds adjacent to Hawaiian pineapple plantings harboring pink pineapples mealybugs. Environmental Entomology, 35: 68-74.

Ramos-Novelo, J. A. 2003. Perspectivas de la red de mango para el 2003. Fideicomisos Instituidos en Relación con la Agricultura (FIRA), pp. 1-13.

Ramos Serrano, J., J. J. Prieto Martínez, O. Rebolledo Domínguez, R. Lezama Gutiérrez, J. R. Sandoval Salazar, J. Velazquez Monreal \& V. H. Salazar Fuentes. 2008. La Guía para la Prevención y Control de Plagas y Enfermedades del Mango, en el Estado de Colima. Gobierno del estado de Colima.

Ranjan, R. 2006. Economic impact of pink hibiscus mealybug in Florida and the United States. Stochastic Environmental Research and Risk Assessment, 20: 353-362.

Rao, V. S. \& S. Srinivasan. 1987. Maconellicoccus hirsutus a new pest of groundnut in Audhra Pradesh. Entomon, 12: 115.

Rhainds, M. \& G. English-Loeb. 2003. Variation in abundance and feeding impact of tarnished plant bug (Hemiptera: Miridae) for different cultivars of strawberry: role of flowering phenology and yield attributes. Journal of Economic Entomology, 96: 433-440.

Roltsch W., D. Meyerdirk, E. Andress, J. Brown, J. Zuniga \& R. Aguilar. 2004. Pink hibiscus mealybug biological control in Imperial Valley, California, 2003 update, pp. 1-4. In: Woods, D.M. (Ed). Biological Control Program Annual Summary, 2003. California Department of Food and Agriculture, Plant Health and Pest Prevention Services, Sacramento, California.

SAGARPA (Secretaria de Agricultura, Ganadería, Desarrollo Rural, Pesca y Alimentación). 2004. Boletín informativo: Campañas de prevención.

SAS, 2001. SAS/STAT User's Guide (Release 8.20). SAS Inst. Inc. Cary, NC, USA.

Sosa, O. 2001. XIV Reunión Extraordinaria de la Comisión técnica del H. CIRSA. 3-4 octubre 2001. Tegucigalpa, Honduras.

Vazquez, L. 1997. Contribución al conocimiento de la chinche harinosa rosada (Maconellicoccus hirsutus Green). Instituto de Investigaciones de Sanidad Vegetal, Boletín Técnico No. 4. Cuba.

Vitullo, J., A. Zhang, C. Mannion \& C. Bergh. 2009. Expression of feeding symptoms from pink hibiscus mealybug (Hemiptera: Pseudococcidae) by commercially important cultivars of hibiscus. Florida Entomologist, 92: 248- 254.

Williams, D. J. 1986. The identity and distribution of the genus Maconellicoccus Ezzat (Hemiptera: Pseudococcidae) in Africa. Bulletin of Entomological Research, 76: 351-357.

Williams, D. J. 1996. A brief account of the hibiscus mealybug, Maconellicoccus hirsutus (Hemiptera: Pseudococcidae), a pest of agriculture and horticulture, with descriptions of two related species from Southern Asia. Bulletin of Entomological Research, 86: 617-628. 